\title{
Solar surface magnetoconvection simulations: A brief review of solar dermatology
}

\author{
CAMERON Robert \\ Max-Planck-Institute für Sonnensystemforschung Katlenburg-Lindau, Germany (email: cameron@mps.mpg.de) \\ Rapid improvement in the speed of computers has enabled realistic simulations of magnetoconvec- \\ tive processes in the surface layers of the Sun. The simulations now cover a wide range of features \\ found in observations. In many cases a direct comparison with the observations is justified, and in all \\ cases our understanding and interpretation of the observations is being improved. Current and future \\ opportunities and difficulties will be discussed.
}

Sun, photosphere, magnetohydrodynamics

In astrophysics there are three routes to progress: observation, simulation, and theory. Simulations are in some sense a two-way bridge between observations and theory: They allow us to test theories by calculating their observational consequences, as well as to interpret observations in terms of usually quite detailed theories. In this paper we will focus on simulations near the solar photosphere - the surface of the Sun - because we now have the computational resources to simulate this region with sufficient verisimilitude to allow a direct comparison with the best available observations.

One method by which progress in this type of simulation can be measured is shown in Figure 1. The figure is an observation of the sun from the KIS/VTT (Tenerife), as processed by Markus Sailer of Göttingen University. Some of the features which have been simulated by various groups are shown in boxes - the quiet Sun, plage, flux emergence, pores, light bridges, the inner penumbra, and the umbrae of sunspots including umbral dots. This image is an update of that shown, for example, at the 2006 IAU symposium on Convection in Astrophysics ${ }^{[1]}$. It is noteworthy that the coverage of features is now more complete, and indeed simulations of entire regions similar to Figure 1 are now being made $^{[2]}$.

"Solar dermatology" is a phrase which partially captures the intent of these types of simulations, namely to try to understand the visible face of the Sun. This layer is itself an important laboratory in which we can explore magnetohydrodynamic process under a wide range of conditions: It is also the layer through which we must deduce what is happening in the deeper layers of the sun. Except for neutrinos, almost all of the information we have concerning what happens inside the Sun has been obtained from photospheric observations.

One of the first magnetoconvective simulations ${ }^{[3]}$ was aimed at elucidating the basic processes, balances, and timescales in a very idealized situation, e.g. 2-D, Bousinesq and without radiative 


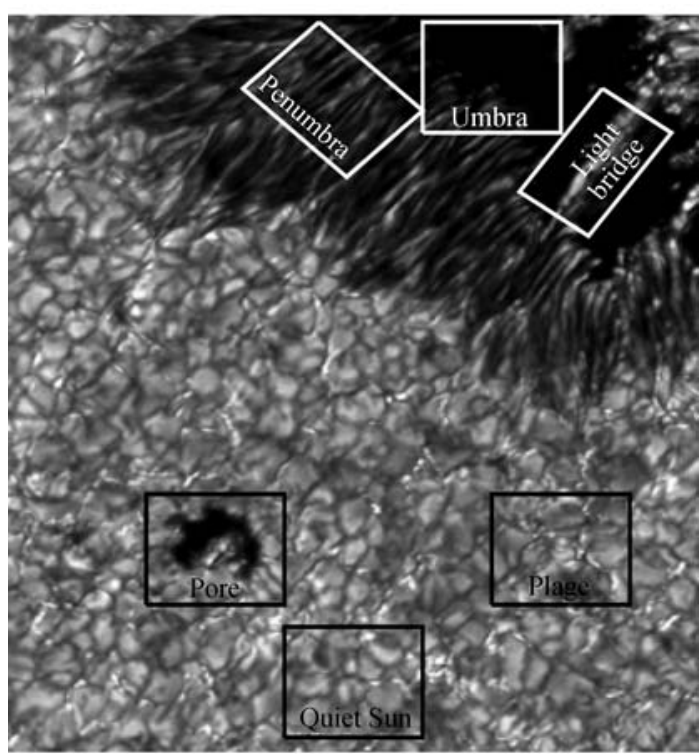

Figure 1 An image from the KIS/VTT Obs. del Teide, Tenerife (in this form courtesy Dr Sailer), of small part of the photosphere showing a variety of structures which have been simulated. Not indicated are regions of flux-emergence.

transfer. These simplifications were necessary in order to make the problem tractable on the computers then available. Axisymmetric geometries ${ }^{[4]}$ could also be treated. Idealized simulations of this type are still being carried out as they continue to improve our understanding of basic processes ${ }^{[5,6]}$.

As a result of Moore's Law new possibilities became available: Simplifications were progressively relaxed. Anelastic magnetoconvection simulations relaxed some of the Bousinesq assumptions, fully compressible treatments became available ${ }^{[7]}$ and finally the effects of radiation (using multiple opacity bins) and partial ionization were introduced ${ }^{[8]}$. Again we note that there still remains much to be learned from the study of topics at each level of realism. In this paper we shall however concentrate on the most realistic simulations of the photospheric layer.

\section{The physics of the photosphere}

The photosphere is the layer of the Sun where the energy transport changes from convective below to radiative above. This transition in transport mechanisms happens because the opacity, being a strong function of temperature and density, falls very rapidly (the density scale height is approximately $100 \mathrm{~km}$ ) so that the photons can finally escape outwards. Thus two essential ingredients for modeling the photosphere are radiative transfer and the inclusion of multiple scale heights. Below the surface the energy transport is via convection so we need the equations of hydrodynamics and an equation of state. For the conditions applicable near the solar surface, the change in ionization levels of various species is important, and these effects must be included in the equation of state. Finally it is an observed fact that magnetic field concentrations of various sizes and strengths can be found on the surface. Part of our aim is to understand these features, and thus we need to deal with magnetohydrodynamics instead of the (somewhat) simpler hydrodynamics. The governing equations can be found, for example, in ref. [9].

\section{Results from simulations}

There has been rapid progress, and a comprehensive summary of solar-surface magnetoconvection simulations is beyond the scope of this paper. Instead we focus on a (somewhat biased) selection of recent results with the aim of giving the flavor of current research and directions for the future.

\subsection{The quiet-Sun: Flux cancellation}

The basic results concerning the nonmagnetic solar atmosphere were obtained in the mid to late 1980 s, for some early results see for example ${ }^{[10,11]}$. This work was rapidly extended to studies of the structure of flux-tubes and helioseismology. An issue under current discussion is how magnetic flux is dispersed and removed from the solar surface.

To study this issue we ${ }^{[12]}$ have performed a series of simulations with different magnetic field initial conditions and have studied how the field decays. Convection in a $6 \mathrm{Mm} \times 6 \mathrm{Mm} \times 1.4 \mathrm{Mm}$ box is allowed to evolve until it reaches a quasi-stationary state. A vertical magnetic field is then imposed. The magnetic field strength is $\pm 200 \mathrm{G}$. The field switches sign in either a $2 \times 2$ or $2 \times 1$ pattern, as is indicated in Figure 2. For both geometries, there is an initial transient where the magnetic energy 


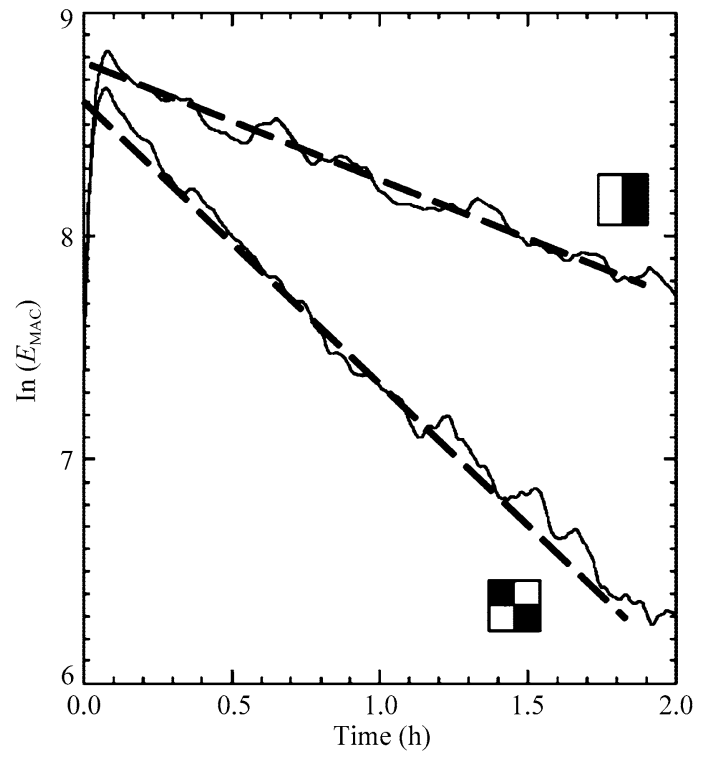

Figure 2 The behavior of the energy in the magnetic field as a function of time for two initial magnetic field distributions.

increases - this is the phase during which the magnetic field is expelled from the interior of granules to the intergranular lanes. After this transient, the field decays over a period of hours. The decay rate is a function of the initial geometry. The e-folding time is about $30 \mathrm{~min}$ for the $2 \times 2$ initial condition. This timescale can be understood in terms of a random walk of magnetic elements residing in the intergranular lanes: The relevant parameters for the random walk are the typical granular size

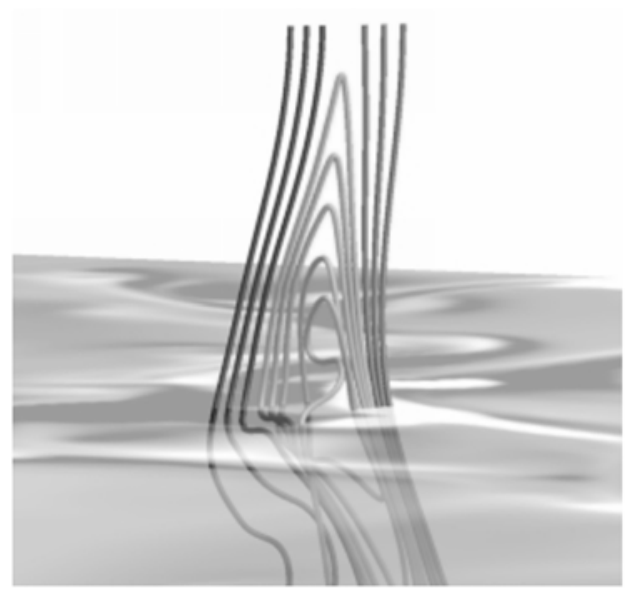

(1 Mm), and the typical granular lifetime (5-10 min). The decay rate of the energy depends upon the longest wavelength mode which contains substantial energy as it is this mode that decays most slowly. This accounts for the different decay rates between the two different initial conditions.

The analysis of the decay rate is based upon the global properties of the field and the rate at which opposite polarity magnetic elements are brought together. In order for magnetic field lines to be removed from the system, a change in topology needs to take place, and individual reconnection events can be identified and analyzed. Figure 3 shows an example of such an event. We comment that the timescale of the reconnection events is small compared to the timescale at which opposite-polarity flux elements are brought together.

\subsection{The quiet-Sun: Dynamo action}

The previous section has described how an imposed magnetic field decays. Provided the magnetic diffusivity is sufficiently small it is possible for a weak magnetic field to be amplified by dynamo action in the photosphere ${ }^{[13]}$. Prior to these simulations it was known that dynamo action could be driven by convection in closed domains ${ }^{[14]}$, but it was not clear what would happen on the Sun where the high degree of stratification means the magnetic field is quickly advected out of the most dynamic regions near the solar surface ${ }^{[15]}$.

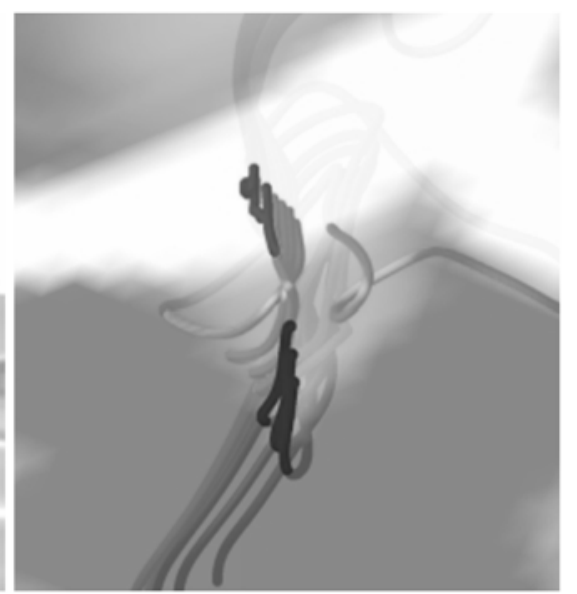

Figure 3 A reconnection event from the simulation of the decaying magnetic field in the quiet Sun. The greyscale shows the vertical component of the magnetic field at a height near the solar surface, whilst the lines show individual fieldlines. Fieldlines with opposite polarities have been pushed together and inverse U-loops can be seen. These loops retract, removing flux from the solar surface. 
The magnetic fields generated by the dynamo (see Figure 4) are likely to be important in understanding both the observations of the quiet sun and possibly for the propagation of energy into the chromosphere.

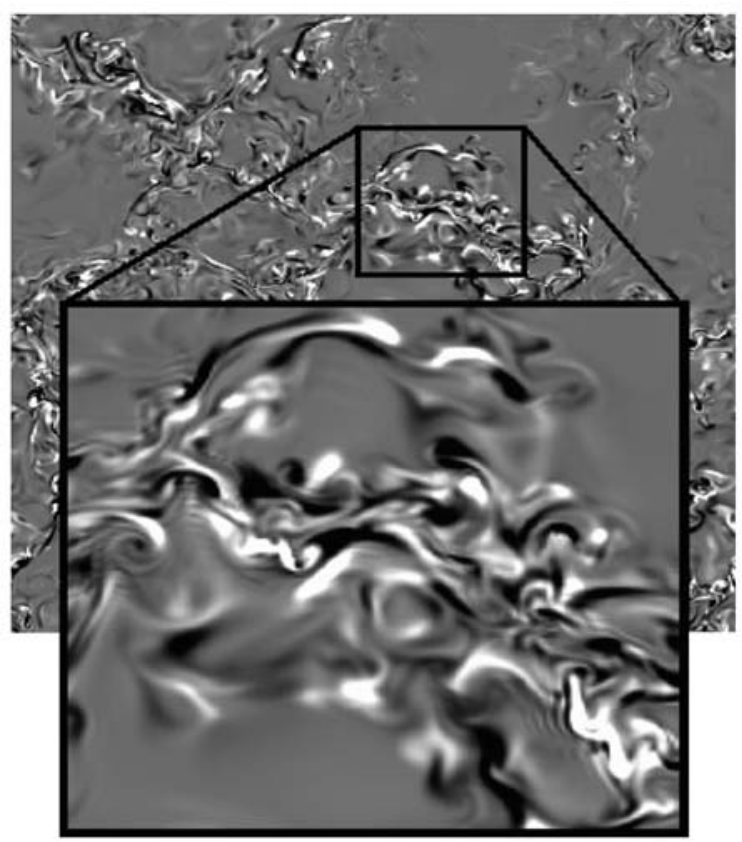

Figure 4 The vertical component of the magnetic field near the surface in a dynamo run. Noticeable is that the field is ubiquitous but highly structured. Most of this structure is hidden from current observations owing to its small horizontal scales ${ }^{[16]}$.

\subsection{Activity: Flux emergence, pores and sunspots}

The quiet Sun covers most of the solar surface, however much interest is focused on the active Sun. Here simulations allow us to understand both observations and the underlying physics. A good example in this context is flux emergence, which has been studied using simulations by Cheung et al. ${ }^{[17]}$. Similarly pores, which are the smallest features clearly associated with the solar cycle have been studied and compared to observations ${ }^{[18]}$. More recently sunspots have been tackled, including sunspot umbrae and umbral dots ${ }^{[19]}$, light bridges $^{[20]}$, and penumbral filaments ${ }^{[21,22]}$. Figure 5 is an image from the simulation by Rempel et al. ${ }^{[22]}$, showing a sunspot umbra including umbral dots, a rudimentary penumbra as well as the quietSun. More recent simulations are even more impressive and include complete spots.

\section{Challenges for today and for the future}

The work sketched above is only a small sample of what is being done in terms of realistic simulations of the photosphere. Clearly the field is progressing rapidly. We are now better able to understand observations of the quiet-Sun, where the surface dynamo plays an important role, and of the penumbral filaments which were open mysteries until recently. Much remains to be done. In the quiet Sun the dependence of the results on the diffusivities, which are much larger in the simulations than in the Sun, is an important issue. For the active Sun, much will be learned by considering the dependence of the results on the imposed initial conditions.

Above the photosphere lies the chromosphere, which involves new challenges - primarily the fact that Local Thermal Equilibrium (LTE) cannot be assumed. Relaxing this assumption enormously increases the computational workload. Progress is however being made ${ }^{[23,24]}$ and increasing computational power, which has powered the field since the 1960's, will continue to accelerate progress in this field.

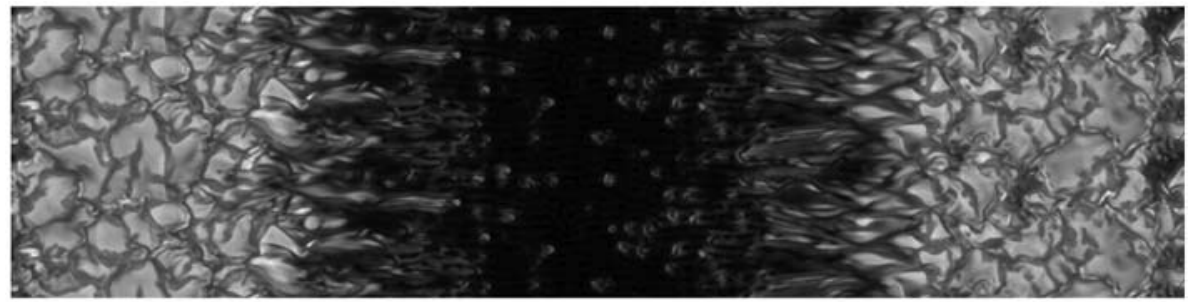

Figure 5 An intensity image from a calculation designed to study penumbral filaments ${ }^{[22]}$. The simulation encompasses quiet Sun, penumbral and umbral regions. 
1 Cameron R, Vögler A, Schüssler M. Photospheric magnetoconvevtion. In: Kupka F, Roxburgh I, Chan K, eds. Convection in Astrophysics, IAU proceedings 239. Cambridge: Cambridge University Press, 2007. 475-481

2 Rempel M, Schüssler M, Cameron R, et al. Penumbral structure and outflows in simulated sunspots. Science, 2009, 325: 171-1174

3 Meyer F, Schmidt H, Wilson P, et al. The growth and decay of sunspots. Mon Not Roy Astron Soc, 1974, 169: 35-37

4 Galloway D J, Moore D R. Axisymmetric convection in the presence of a magnetic field. Geophys Astrophys Fluid Dyn, 1979, 12: 73-105

5 Cameron R, Galloway D J. The structure of small-scale magnetic flux tubes. Mon Not Roy Astron Soc, 2005, 358: 10251035

6 Bushby P J, Houghton S M, Proctor M R E, et al. Convective intensification of magnetic fields in the quiet Sun. Mon Not Roy Astron Soc, 2008, 387: 698-706

7 Graham E. Numerical simulation of two-dimensional compressible convection. J Fluid Mech, 1975, 70: 689-703

8 Nordlund $\AA$. Numerical simulations of the solar granulation. I- Basic equations and methods. Astron Astrophys, 1982, 107: 1-10

9 Vögler A, Shelyag S, Schüssler M, et al. Simulations of magneto-convection in the solar photosphere. Equations, methods, and results of the MURaM code. Astron Astrophys, 2005, 429: $335-351$

10 Nordlund Å. Solar convection. Sol Phys, 1985, 100: 209-235

11 Stein R F, Nordlund A. Topology of convection beneath the solar surface. Astrophys J, 1989, 342: L95-L98

12 Vögler A, Cameron R, Schüssler M. (in preparation)

13 Vögler A, Schüssler M. A solar surface dynamo. Astron Astrophys, 2007, 465: L43-L46

14 Cattaneo F. On the origin of magnetic fields in the quiet photosphere. Astrophys J, 1999, 515: L39-L42
15 Stein R F, Bercik D, Nordlund Å. Solar Surface MagnetoConvection. In: Pevtsov A, Uitenbroek H, eds. Current theoretical models and future high resolution solar observations: preparing for ATST. Astron Soc Pac Conf, 2003, 286: 121131

16 Pietarila Graham J, Danilovic S, Schüssler M. Turbulent magnetic fields in the quiet Sun: Implications of hinode observations and small-scale dynamo simulations. Astrophys J, 2009, 693: 1728-1735

17 Cheung M, Schüssler M, Moreno-Insertis F. Magnetic flux emergence in granular convection: Radiative MHD simulations and observational signatures. Astron Astrophys, 2007, 467: 703-719

18 Cameron R, Schüssler M, Vögler A, et al. Radiative magnetohydrodynamic simulations of solar pores. Astron Astrophys, 2007, 474: 261-272

19 Schüssler M, Vögler A. Magnetoconvection in a Sunspot Umbra. Astrophys J, 2006, 641: L73-L76

20 Nordlund $\AA$. The Future: Where are We Headed? In: Leibacher J, Stein R F, Uitenbroek H, eds. Solar MHD theory and observations: A high spatial resolution perspective. Astron Soc Pac Conf, 2006, 354: 353-364

21 Heinemann T, Nordlund A, Scharmer G B, et al. MHD simulations of penumbra fine structure. Astrophys J, 2007, 669: 1390-1394

22 Rempel M, Schüssler M, Knölker M. Radiative magnetohydrodynamic simulation of sunspot structure. Astrophys J, 2009, 691: 640-649

23 Leenaarts J, Wedemeyer-Böhm S. Time-dependent hydrogen ionisation in 3D simulations of the solar chromosphere. Methods and first results. Astron Astrophys, 2006, 460: 301-307

24 Hansteen V, Carlsson M, Gudiksen B. 3D numerical models of the chromosphere, transition region, and corona. In: Heinzel P, Dorotovič I, Rutten R J, eds. The Physics of Chromospheric Plasmas. Astron Soc Pac Conf, 2007, 368: 107-114 